\title{
PROBABILITY MEASURES ON SEMIGROUPS
}

\author{
PETER GERL
}

\begin{abstract}
Let $S$ be a discrete semigroup, $P$ a probability measure on $S$ and $s \in S$ with $\lim \sup _{n}\left(P^{(n)}(s)\right)^{1 / n}=1$. We study limit theorems for the convolution powers $P^{(n)}$ of $P$ implied by the above property and further the class of all semigroups with this property. Theorem 3 relates this class of semigroups to left amenable semigroups.
\end{abstract}

1. Introduction. Let $S$ be a discrete semigroup and $P$ a probability measure on $S$, that is a real valued function on $S$ with $P(s) \geqq 0$ for all $s \in S$ and $\sum_{s \in S} P(s)=1$. Kesten ([4], [5]) characterized amenable groups by means of the asymptotic behavior of convolution powers of symmetric probability measures defined on the group. A more precise information for the asymptotic behavior was obtained in [2] and [3] for symmetric probability measures on a discrete amenable group. In what follows we will derive similar theorems for probability measures on discrete semigroups.

Let $S$ be a discrete semigroup, $P$ a probability measure on $S$. Then Supp $P=\{s / P(s)>0\}$ denotes the support of $P$. To say Supp $P$ generates $S$ means: $S=\bigcup_{n=1}^{\infty}(\operatorname{Supp} P)^{n}$.

For probability measures $P, Q$ on $S$ define their convolution $P * Q$ by

$$
P * Q(s)=\sum_{s_{1} s_{2}=s} P\left(s_{1}\right) Q\left(s_{2}\right)
$$

(the summation is to be extended over all representations of $s$ as a product of two elements $s_{1}, s_{2}$ of $S$ ). $P * Q$ is again a probability measure and Supp $P * Q=(\operatorname{Supp} P) \cdot(\operatorname{Supp} Q)$. We often write $P^{(1)}=P, P\left({ }^{n}\right)=P * P^{(n-1)}$.

Kesten obtained the following characterization of discrete amenable groups:

Let $G$ be a discrete group with unit element $e, P$ a symmetric probability measure on $G\left(P(g)=P\left(g^{-1}\right)\right.$ for all $g$ in $\left.G\right)$ such that $G$ is generated by

Received by the editors January 2, 1973.

AMS (MOS) subject classifications (1970). Primary 43A05, 43A07; Secondary 20M10, 60B15, 60F99.

Key words and phrases. Semigroup, probability measure, convolution, limit theorems, amenable semigroup.

(c) American Mathematical Society 1973 
Supp $P$; then

$$
G \text { is amenable } \Leftrightarrow P[e]=\limsup _{n \rightarrow \infty}\left(P^{(n)}(e)\right)^{1 / n}=1 .
$$

2. Limit theorems. Let $S$ be a discrete semigroup; if there exists a probability measure $P$ on $S$ with the properties

(1) Supp $P$ generates $S$, and

(2) there exists an $s \in S$ with $P[s]=\lim \sup _{n \rightarrow \infty}\left(P^{(n)}(s)\right)^{1 / n}=1$ then we call $S$ an $A$-semigroup or, if we want to specify $P,(S, P)$ an $A$-pair.

Let $(S, P)$ be an $A$-pair with $P[s]=1$ for some $s \in S$. Let $s^{\prime} \in S$; since Supp $P$ generates $S$ there exists a natural number $k$ with $P^{(k)}\left(s^{\prime}\right)>0$. Then

and ( $k$ is fixed)

$$
P^{(n+k)}\left(s s^{\prime}\right)=\sum_{s_{1} s_{2}=s s^{\prime}} P^{(n)}\left(s_{1}\right) P^{(k)}\left(s_{2}\right) \geqq P^{(n)}(s) P^{(k)}\left(s^{\prime}\right)
$$

$$
\begin{aligned}
1 \geqq P\left[s s^{\prime}\right] & =\lim \sup _{n}\left(P^{(n+k)}\left(s s^{\prime}\right)\right)^{1 /(n+k)} \\
& \geqq \lim _{n} \sup \left(P^{(n)}(s)\right)^{1 / n} \lim _{n}\left(P^{(k)}\left(s^{\prime}\right)\right)^{1 / n}=P[s]=1 .
\end{aligned}
$$

Therefore we have

Proposition 1. $P[s]=1 \Rightarrow P\left[s s^{\prime}\right]=P\left[s^{\prime} s\right]=P\left[s^{\prime} s s^{\prime \prime}\right]=1$ for all $s^{\prime}, s^{\prime \prime} \in S$.

$S$ is called left simple if for all $s \in S: S s=S$ (this means every element of $S$ can be written in the form $s^{\prime} s$ ). Proposition 1 implies

Proposition 2. (a) If $S$ is left simple (or right simple, or a group) then

$$
P[s]=1 \text { for one } s \in S \Leftrightarrow P[s]=1 \text { for every } s \in S .
$$

(b) If $S$ has a left unit e (es $=s$ for all $s$ ) then

$$
P[e]=1 \Leftrightarrow P[s]=1 \text { for every } s \in S .
$$

Let $S$ be a discrete semigroup with a left unit $e$ and $(S, P)$ an $A$-pair, further put $P^{\prime}=\frac{1}{2}\left(P+\delta_{e}\right) \quad\left(\delta_{e}\right.$ is the probability measure concentrated at $e$, i.e. $\delta_{e}(e)=1, \delta_{e}(s)=0$ for $\left.e \neq s \in S\right)$. Then $P^{\prime}$ is a probability measure on $S$ and Supp $P^{\prime}=\operatorname{Supp} P \cup\{e\}$.

Proposition 3. $P[s]=1 \Rightarrow P^{\prime}[s]=1$.

Proof. $e$ is a left unit, therefore $\delta_{e} * P=P$. So

and

$$
P^{\prime(2 n)}(s) \geqq \frac{1}{2^{2 n}} \sum_{k=1}^{2 n} \frac{1}{2}\left(\begin{array}{c}
2 n \\
k
\end{array}\right) P^{(k)}(s) \geqq \frac{1}{4^{n}} \frac{1}{2}\left(\begin{array}{c}
2 n \\
n
\end{array}\right) P^{(n)}(s)
$$

$$
1 \geqq\left(P^{\prime(2 n)}(s)\right)^{1 / 2 n} \geqq \frac{1}{2}\left(\frac{1}{2}\right)^{1 / 2 n}\left(\begin{array}{c}
2 n \\
n
\end{array}\right)^{1 / 2 n}\left(P^{(n)}(s)\right)^{1 / 2 n}=a_{n}\left(P^{(n)}(s)\right)^{1 / 2 n}
$$

Since $\lim _{n} a_{n}=1$ we get $P^{\prime}[s]=1$. 
Proposition 3 says that if $(S, P)$ is an $A$-pair then so is $\left(S, P^{\prime}\right)$ (and we have $\left.P^{\prime}(e)>0\right)$.

THEOREM 1. Let $S$ be a discrete semigroup with a left unit e and $(S, P)$ an A-pair. Then

(1) $P[e]=1 \Rightarrow \lim _{n \rightarrow \infty}\left(P^{\prime(n)}(s)\right)^{1 / n}=1$ for every $s \in S$,

(2) $\lim _{n \rightarrow \infty}\left(P^{\prime(n)}(s)\right)^{1 / n}=1 \Leftrightarrow \lim _{n \rightarrow \infty}\left(P^{\prime(n+1)}(s) / P^{\prime(n)}(s)\right)=1$.

Proof. Similar to the proof of Theorem 1 and Theorem 2 of [3].

3. The class of $A$-semigroups.

THEOREM 2. Let $S$ be a finite semigroup and $P$ a probability measure on $S$ such that Supp $P$ generates $S$. Then $(S, P)$ is an A-pair.

Proof. Let $c$ be the cardinal number of $S$. Then for every $n=1,2, \cdots$ there exists an element $s_{n}$ in $S$ with $P^{(n)}\left(s_{n}\right) \geqq 1 / c$. Because $S$ is finite there is an $s_{0}$ in $S$ which appears infinitely often in the sequence $s_{1}, s_{2}, \cdots$ and so $P^{\left(n_{k}\right)}\left(s_{0}\right) \geqq 1 / c$ for some sequence $n_{1}<n_{2}<\cdots$ of natural numbers. Therefore $P\left[s_{0}\right]=1$.

THEOREM 3. Let $S$ be a discrete semigroup with left cancellation $\left(s s^{\prime}=s s^{\prime \prime} \Rightarrow s^{\prime}=s^{\prime \prime}\right)$ and a left unit e. If $S$ is an A-semigroup then $S$ is left amenable.

Proof. By assumption there exists a probability measure $P$ on $S$ and an element $s \in S$ such that (1) Supp $P$ generates $S$ and (2) $P[s]=1$. By Proposition 3 we have $P^{\prime}[s]=1\left(P^{\prime}=\frac{1}{2}\left(P+\delta_{e}\right)\right)$.

Now let $x \in l_{2}(S)$; then $P^{\prime} * x \in l_{2}(S)$ and $\left\|P^{\prime} * x\right\|_{2} \leqq\left\|P^{\prime}\right\|_{1}\|x\|_{2}=\|x\|_{2}$. So we can consider $P^{\prime} *$ as an operator on $l_{2}(S)$ and we have for its norm $\left\|P^{\prime} *\right\|_{2 \rightarrow 2} \leqq 1$.

Further, $\delta_{e} \in l_{2}(S)$. Next,

$$
\begin{aligned}
P^{\prime}(s) & =P^{\prime}(s) \delta_{e}(e) \leqq\left(\sum_{s \in S}\left(\sum_{s_{1} s_{2}=s} P^{\prime}\left(s_{1}\right) \delta_{e}\left(s_{2}\right)\right)^{2}\right)^{1 / 2}=\left\|P^{\prime} * \delta_{e}\right\|_{2} \\
& \leqq \sup _{\|x\|_{2}=1}\left\|P^{\prime} * x\right\|_{2}=\left\|P^{\prime} *\right\|_{2 \rightarrow 2} \leqq 1,
\end{aligned}
$$

and in the same way

$$
P^{\prime(n)}(s) \leqq\left\|P^{\prime(n)} *\right\|_{2 \rightarrow 2} \leqq 1 .
$$

So $1=P^{\prime}[s] \leqq \lim \sup _{n}\left\|P^{\prime(n)} *\right\|_{2 \rightarrow 2}^{1 / n}=$ spectral radius of $P^{\prime} * \leqq\left\|P^{\prime} *\right\|_{2 \rightarrow 2} \leqq 1$ or $\left\|P^{\prime} *\right\|_{2 \rightarrow 2}=1$; by the same argument $\left\|P^{\prime(k)} *\right\|_{2 \rightarrow 2}=1$ for $k=1,2, \cdots$. But Supp $P^{\prime}$ generates $S$ and so for every finite $E \subset S$ there exists a natural number $k$ with $E \subset \operatorname{Supp} P^{\prime(k)}$ and $e \in \operatorname{Supp} P^{\prime(k)}$. Then [1] (Theorem 1, (e) $\Rightarrow($ a)) implies that $S$ is left amenable.

REMARK 1. For $S$ a group $G$ and $P$ a symmetric probability measure on $G$ whose support generates $G$ we have from the theorem of Kesten 
and Proposition 2: $G$ amenable $\Rightarrow P[g]=1$ for every $g \in G$. So for groups we lose nothing in considering only symmetric probability measures. If $P$ is not symmetric this implication need no longer be true; consider for example the infinite cyclic group $G=\langle a\rangle$, generated by $a$. This group is commutative, therefore amenable. Let

Then

$$
P=\alpha \delta_{a}+(1-\alpha) \delta_{a^{-1}} \quad\left(0<\alpha<1, \alpha \neq \frac{1}{2}\right) .
$$

and

$$
P^{(n)}=\sum_{k=0}^{n}\left(\begin{array}{l}
n \\
k
\end{array}\right) \delta_{a^{2 k-n} \alpha^{k}(1-\alpha)^{n-k}}
$$

So

$$
P^{(2 n)}(e)=\left(\begin{array}{c}
2 n \\
n
\end{array}\right) \alpha^{n}(1-\alpha)^{n}
$$

$$
P[e]=\lim _{n \rightarrow \infty}\left(\begin{array}{c}
2 n \\
n
\end{array}\right)^{1 / 2 n}(\alpha(1-\alpha))^{1 / 2}=2(\alpha(1-\alpha))^{1 / 2}<1 \quad \text { for } \alpha \neq \frac{1}{2}
$$

(and $P[e]=P[g]$ for every $g \in G$ by Proposition 2).

REMARK 2. The statement of Theorem 3 is false for arbitrary semigroups, for there are finite semigroups (which are $A$-semigroups by Theorem 2) that are not left (or right) amenable.

REMARK 3. The converse of Theorem 3 is not true in general, for there are left amenable semigroups with left cancellation and a unit that are not $A$-semigroups.

Consider, for example, the infinite cyclic semigroup $S=\left\{e, a, a^{2}, \cdots\right\}$, generated by $e$ (unit) and $a$. $S$ is abelian and therefore amenable. Let $P$ be a probability measure on $S$ such that Supp $P$ generates $S$. This implies $0<P(e)=\alpha<1$ and so $P=\alpha \delta_{e}+(1-\alpha) P_{1}$, where Supp $P_{1} \subset\left\{a, a^{2}, \cdots\right\}=$ $S-\{e\}$.

Then Supp $P_{1}^{(n)} \subset\left\{a^{n}, a^{n+1}, \cdots\right\}$ and therefore

$$
P^{(n)}=\sum_{k=0}^{n}\left(\begin{array}{l}
n \\
k
\end{array}\right) P_{1}^{(k)} \alpha^{n-k}(1-\alpha)^{k} .
$$

This gives $P^{(n)}(e)=\alpha^{n}$ and $P[e]=\alpha<1$.

For $l=1,2, \cdots$ we find for $n$ large enough

$$
\begin{aligned}
P^{(n)}\left(a^{l}\right) & \leqq \sum_{k=0}^{l}\left(\begin{array}{l}
n \\
k
\end{array}\right) P_{1}^{(k)}\left(a^{l}\right) \alpha^{n-k}(1-\alpha)^{k} \\
& \leqq \sum_{k=0}^{l}\left(\begin{array}{l}
n \\
k
\end{array}\right) \alpha^{n-k}(1-\alpha)^{k} \leqq \alpha^{n}\left(\begin{array}{l}
n \\
l
\end{array}\right) \sum_{k=0}^{l}\left(\frac{1-\alpha}{\alpha}\right)^{k}
\end{aligned}
$$

and therefore $P\left[a^{l}\right] \leqq \alpha<1$. So $S$ is not an $A$-semigroup. 
TheOREM 4. The homomorphic image of an A-semigroup is an $A$ semigroup.

Proof. Let $(S, P)$ be an $A$-pair with $P[s]=1 \quad(s \in S)$. Let $\varphi: S \rightarrow S_{1}$ be a homomorphism onto the semigroup $S_{1}$. Define the probability measure $P_{1}$ on $S_{1}$ by

Then by induction

$$
P_{1}\left(s_{1}\right)=P\left(\varphi^{-1}\left(s_{1}\right)\right)=\sum_{s \in \varphi^{-1}\left(s_{1}\right)} P(s) .
$$

$$
\begin{aligned}
P_{1}^{(n)}\left(s_{1}\right) & =\sum_{s_{1}^{\prime} s_{1}^{\prime \prime}=s_{1}} \sum_{s^{\prime} \in \varphi^{-1}\left(s^{\prime}\right)} P\left(s^{\prime}\right) \sum_{s^{\prime \prime} \in \varphi^{-1}\left(s_{1}^{\prime \prime}\right)} P^{(n-1)}\left(s^{\prime \prime}\right) \\
& =\sum_{s^{\prime} s^{\prime \prime} \in \varphi^{-1}\left(s_{1}\right)} P\left(s^{\prime}\right) P^{(n-1)}\left(s^{\prime \prime}\right)=\sum_{s \in \varphi^{-1}\left(s_{1}\right)} P^{(n)}(s),
\end{aligned}
$$

and therefore $1 \geqq P_{1}^{(n)}\left(s_{1}\right) \geqq P^{(n)}(s)$ for $s \in \varphi^{-1}\left(s_{1}\right)$. Thus $P_{1}\left[s_{1}\right]=1$ if $P[s]=1\left(\right.$ where $\left.\varphi(s)=s_{1}\right)$.

Theorem 5. Let $\left(S_{1}, P_{1}\right)$ be an A-pair, $\left(S_{2}, P_{2}\right)$ be an A-pair such that for some $s_{2} \in S_{2}: \lim _{n \rightarrow \infty}\left(P_{2}^{(n)}\left(s_{2}\right)\right)^{1 / n}=1$. Then $\left(S_{1} \times S_{2}, P_{1} \times P_{2}\right)$ is an A-pair.

Proof. Supp $P_{1} \times P_{2}$ generates $S_{1} \times S_{2}$ and

$$
1 \geqq\left(P_{1} \times P_{2}\right)\left[\left(s_{1}, s_{2}\right)\right] \geqq P_{1}\left[s_{1}\right] \lim \left(P_{2}^{(n)}\left(s_{2}\right)\right)^{1 / n}=P_{1}\left[s_{1}\right]=1
$$

for some $s_{1} \in S_{1}$.

EXAMPLE 1. Let $S$ be a countable right zero semigroup $\left(s s^{\prime}=s^{\prime}\right.$ for all $s, s^{\prime} \in S$ ). If $P$ is any probability measure on $S$ whose support generates $S$, then $\operatorname{Supp} P=S$ and

$$
P^{(n)}(s)=\sum_{s_{2}=s_{1} s_{2}=s} P\left(s_{1}\right) P^{(n-1)}\left(s_{2}\right)=\sum_{s_{1} \in S} P\left(s_{1}\right) P^{(n-1)}(s)=P(s) .
$$

Therefore $P[s]=\lim (P(s))^{1 / n}=1$, because $P(s)>0$ for every $s \in S$; so we see that every countable right zero semigroup is an $A$-semigroup.

EXAMPLE 2. As in Remark 3 one can show that the semigroup $S=\{e, a, b, a b, \cdots\}$, generated by two elements $a$ and $b$, is not an $A$-semigroup.

\section{REFERENCES}

1. M. M. Day, Convolutions, means and spectra, Illinois J. Math. 8 (1964), 100-111. MR 28 \#2447.

2. P. Gerl, Über die Anzahl der Darstellungen von Worten, Monatsh. Math. 75 (1971), 205-214.

3. —_ Diskrete, mittelbare Gruppen, Monatsh. Math. (to appear). 
4. H. Kesten, Symmetric random walks on groups, Trans. Amer. Math. Soc. 92 (1959), 336-354. MR 22 \#253.

5. - Full Banach mean values on countable groups, Math. Scand. 7 (1959), 146-156. MR 22 \#2911.

Department of Mathfmatics, Pahlavi University, Shiraz, Iran

Current address: Mathematisches Institut der Universität, Strudlhofgasse 4, A-1090 Wien, Austria 